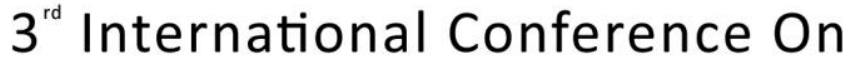 Advanced Research in EDUCATION \\ 11-14 March, $2021 \quad$ Oxford - United Kingdom
}

\section{Exploring The Application of Neural Networks and Transfer Learning in Predicting Post-Secondary Student Outcomes}

\author{
Karthik Vetrivel \\ Formerly the University of Washington, Bothell, USA \\ kvetriv@uw.edu
}

\begin{abstract}
Driven to make more informed decisions, educational technology researchers have recently turned towards blending machine learning and educational data mining to predict student outcomes. In this study, we have identified and cleaned a National Center for Education Statistics longitudinal dataset containing 23,000 high school students' baseline variables (race, income, GPA, etc.) and their postsecondary outcomes (college matriculation). We applied two machine learning techniques--neural networks and transfer learning--on this 9-year dataset and evaluated each model's relative performance in terms of recall and precision to successfully predict student outcomes. Our goal was to see if neural transfer learning could reasonably be used to train new predictive models compared to novel baseline neural networks in an educational context. Outcomes of this report provide an insight into how education technology researchers can further develop transfer learning in education, specifically in the context of the HSLS:09 datasets.
\end{abstract}

Keywords: NCES; neural networks; neural transfer learning; predictive analytics; educational data $\operatorname{mining}$ 